\title{
A Note on Normal Families of Meromorphic Functions Concerning Shared Values
}

\author{
Yuan Wenjun, ${ }^{1}$ Wei Jinjin, ${ }^{1}$ and Lin Jianming ${ }^{2}$ \\ ${ }^{1}$ School of Mathematics and Information Science, Guangzhou University, Guangzhou 510006, China \\ 2 School of Economy and Management, Guangzhou University of Chinese Medicine, \\ Guangzhou 510006, China
}

Correspondence should be addressed to Wei Jinjin, weijinjingirl@163.com and Lin Jianming, ljmguanli@21cn.com

Received 2 July 2011; Accepted 26 August 2011

Academic Editor: Cengiz Çinar

Copyright (C) 2011 Yuan Wenjun et al. This is an open access article distributed under the Creative Commons Attribution License, which permits unrestricted use, distribution, and reproduction in any medium, provided the original work is properly cited.

\begin{abstract}
We study the normality of families of meromorphic functions related to a Hayman conjecture. We consider whether a family of meromorphic functions $\mathcal{F}$ is normal in $D$ if, for every pair of functions $f$ and $g$ in $\mathcal{F}, f^{\prime}-a f^{n}$ and $g^{\prime}-a g^{n}$ share the value $b$ for $n=1,2$, and 3 , where $a$ and $b \neq 0$ are two finite complex numbers. Some examples show that the conditions in our results are the best possible.
\end{abstract}

\section{Introduction and Main Results}

Let $f(z)$ and $g(z)$ be two nonconstant meromorphic functions in a domain $D \subseteq \mathbb{C}$, and let $a$ be a finite complex value. We say that $f$ and $g$ share $a \mathrm{CM}$ (or IM) in $D$ provided that $f-a$ and $g-a$ have the same zeros counting (or ignoring) multiplicity in $D$. When $a=\infty$ the zeros of $f-a$ mean the poles of $f$ (see [1]). It is assumed that the reader is familiar with the standard notations and the basic results of Nevanlinna's value-distribution theory ([2-4] or $[1])$.

Bloch's principle [5] states that every condition which reduces a meromorphic function in the plane $\mathbb{C}$ to be a constant forces a family of meromorphic functions in a domain $D$ normal. Although the principle is false in general (see [6]), many authors proved the normality criterion for families of meromorphic functions corresponding to Liouville-Picardtype theorem (see [7] or [4]).

It is also more interesting to find normality criteria from the point of view of shared values. In this area, Schwick [8] first proved an interesting result that a family of meromorphic functions in a domain is normal if it in every function in it shares three distinct 
finite complex numbers with its first derivative. Later, more results about normality criteria concerning shared values can be found, for instance, see [9-11] and so on. In recent years, this subject has attracted the attention of many researchers worldwide. [12].

We now first introduce a normality criterion related to a Hayman normal conjecture

Theorem 1.1. Let $\mp$ be a family of holomorphic (meromorphic) functions defined in a domain $D$, $n \in \mathbb{N}, a \neq 0, b \in \mathbb{C}$. If $f^{\prime}(z)+a f^{n}(z)-b \neq 0$ for each function $f(z) \in \mathcal{F}$ and $n \geq 2(n \geq 3)$, then $\mathcal{F}$ is normal in $D$.

The results for the holomorphic case are due to Drasin [7] for $n \geq 3$, Pang [13] for $n=3$, and Chen and Fang [14] for $n=2$, Ye [15] for $n=2$, and Chen and Gu [16] for the generalized result with $a$ and $b$ replaced by meromorphic functions. The results for the meromorphic case are due to Li [17], Li [18], and Langley [19] for $n \geq 5$, Pang [13] for $n=4$, Chen and Fang [14] for $n=3$, and Zalcman [20] for $n=3$, obtained independently.

When $n=2$ and $f$ is meromorphic, Theorem 1.1 is not valid in general. Fang and Yuan [21] gave an example to this, and moreover a result added other conditions below.

Example 1.2. The family of meromorphic functions $\mathcal{F}=\left\{f_{j}(z)=j z /(\sqrt{j} z-1)^{2}: j=1,2, \ldots,\right\}$ is not normal in $D=\{z:|z|<1\}$. This is deduced by $f_{j}^{\#}(0)=j \rightarrow \infty$, as $j \rightarrow \infty$, and Marty's criterion [2], although, for any $f_{j}(z) \in \mathcal{F}, f_{j}^{\prime}+f_{j}^{2}=j(\sqrt{j} z-1)^{-4} \neq 0$.

Here $f^{\#}(\xi)$ denotes the spherical derivative

$$
f^{\#}(\xi)=\frac{\left|f^{\prime}(\xi)\right|}{1+|f(\xi)|^{2}} .
$$

Theorem 1.3. Let $\mathbb{F}$ be a family of meromorphic functions in a domain $D$ and $a \neq 0, b \in \mathbb{C}$. If $f^{\prime}(z)+$ $a(f(z))^{2}-b \neq 0$ and the poles of $f(z)$ are of multiplicity $\geq 3$ for each $f(z) \in \mathcal{F}$, then $\mathcal{F}$ is normal in $D$.

In 2008, by the ideas of shared values, Zhang [11] proved the following.

Theorem 1.4. Let $F$ be a family of meromorphic (holomorphic) functions in $D, n$ a positive integer, and $a, b$ two finite complex numbers such that $a \neq 0$. If $n \geq 4(n \geq 2)$ and, for every pair of functions $f$ and $g$ in $\mathcal{F}, f^{\prime}-a f^{n}$ and $g^{\prime}-a g^{n}$ share the value $b$, then $\mathcal{F}$ is normal in $D$.

Example 1.5 (see [11]). The family of meromorphic functions $\mathcal{F}=\left\{f_{j}(z)=1 /(\sqrt{j}(z-1 / j))\right.$ : $j=1,2, \ldots$,$\} is not normal in D=\{z:|z|<1\}$. Obviously $f_{j}^{\prime}-f_{j}^{3}=-z /\left(\sqrt{j}(z-1 / j)^{3}\right)$. So, for each pair $m, j, f_{j}^{\prime}-f_{j}^{3}$ and $f_{m}^{\prime}-f_{m}^{3}$ share the value 0 in $D$, but $\mathcal{F}$ is not normal at the point $z=0$ since $f_{j}^{\#}(0)=2(\sqrt{j})^{3} / 1+j \rightarrow \infty$, as $j \rightarrow \infty$.

Remark 1.6. Example 1.5 shows that Theorem 1.4 is not valid when $n=3$, and the condition $n=4$ is the best possible for the meromorphic case.

It is natural to ask under what conditions Theorem 1.4 holds for $n \leq 3$. In this paper, we answer the above question and prove the following results. 
Theorem 1.7. Let $\mp$ be a family of meromorphic functions in $D$ and $a$ and $b$ two finite complex numbers such that $a \neq 0$. Suppose that each $f(z) \in \mathcal{F}$ has no simple pole. If $f^{\prime}-a f^{3}$ and $g^{\prime}-a g^{3}$ share the value $b$ for every pair of functions $f$ and $g$ in $\mathcal{F}$, then $\mathcal{F}$ is normal in $D$.

Remark 1.8. Example 1.5 shows that the condition added in Theorem 1.7 about the multiplicity of poles of $f(z)$ is the best possible.

Theorem 1.9. Let $₹$ be a family of meromorphic functions in $D$ and $a$ and $b$ two finite complex numbers such that $a \neq 0$. Suppose that $f(z)$ admits the zeros of multiple and the poles of multiplicity $\geq 3$ for each $f(z) \in \mathcal{F}$. If $f^{\prime}-a f^{2}$ and $g^{\prime}-a g^{2}$ share the value $b$ for every pair of functions $f$ and $g$ in $\mathcal{F}$, then $\mathcal{F}$ is normal in $D$.

Remark 1.10. Example 1.2 shows that the condition added in Theorem 1.9 about the multiplicity of poles and zeros of $f(z)$ is the best possible.

Theorem 1.11. Let $₹$ be a family of meromorphic functions in $D$ and $a$ and $b$ two nonzero finite complex numbers. Suppose that $f(z) \neq 0$ and that its poles are multiple for each $f(z) \in \mathcal{F}$. If $f^{\prime}-$ af and $g^{\prime}-a g$ share the value $b$ for every pair of functions $f$ and $g$ in $\mathcal{F}$, then $F$ is normal in $D$.

Corollary 1.12. Let $\mp$ be a family of holomorphic functions in $D$ and $a$ and $b$ two finite complex numbers such that $b \neq 0$. Suppose that $f(z) \neq 0$ for each $f(z) \in \mathcal{F}$. If $f^{\prime}-a f$ and $g^{\prime}-a g$ share the value $b$ for every pair of functions $f$ and $g$ in $F$, then $F$ is normal in $D$.

Example 1.13. The family of holomorphic functions $\mathcal{F}=\left\{f_{j}(z)=j z e^{z}-j e^{z}+j-b: j=1,2, \ldots,\right\}$ is not normal in $D=\{z:|z|<1\}$. Obviously $f_{j}^{\prime}-f_{j}=j\left(e^{z}-1\right)+b$. So, for each pair $m, j, f_{j}^{\prime}-f_{j}$ and $f_{m}^{\prime}-f_{m}$ share the value $b$ in $D$. On the other hand, $f_{j}(0)=-b, f_{j}(1 / \sqrt{j})=\sqrt{j}(1+(1 / \sqrt{j})+$ $o(1)) \rightarrow \infty$, as $j \rightarrow \infty$. This implies that the family $\mathcal{F}$ fails to be equicontinuous at 0 , and thus $\mathcal{F}$ is not normal at 0 .

Theorem 1.14. Let $\mp$ be a family of meromorphic functions in $D$ and $a$ and $b$ two finite complex numbers such that $b \neq 0$. Suppose that $f(z) \neq 0$ and $f^{\prime}(z)-a f(z) \neq b$ for each $f(z) \in \mathcal{F}$. Then $\mathcal{F}$ is normal in $D$.

Example 1.15. The family of holomorphic functions $\mathscr{F}=\left\{f_{j}(z)=j(z+1)-1: j=1,2, \ldots,\right\}$ is normal in $D=\{z:|z|<1\}$. Obviously $f_{j}(z) \neq 0$ and $f_{j}^{\prime}-f_{j}=-j z+1$. So, for each pair $m, j, f_{j}^{\prime}-f_{j}$ and $f_{m}^{\prime}-f_{m}$ share the value 1 in $D$. Corollary 1.12 implies that the family $\mathcal{F}$ is normal in $D$.

Example 1.16. The family of meromorphic functions $\mathcal{F}=\left\{f_{j}(z)=(z / j)-1: j=1,2, \ldots,\right\}$ is normal in $D=\{z:|z|<1\}$. The reason is that the conditions of Theorem 1.14 hold that $f_{j}(z) \neq 0$ and $f_{j}^{\prime}-f_{j}=((1-z) / j)+1 \neq 1$ in $D=\{z:|z|<1\}$.

Remark 1.17. Example 1.13 shows that Theorem 1.4 is not valid when $n=1$ and in the holomorphic case and the condition $f(z) \neq 0$ is necessary in Theorem 1.11, Corollary 1.12. Both Examples 1.15 and 1.16 tell us that Corollary 1.12 and Theorem 1.14 occur.

\section{Preliminary Lemmas}

In order to prove our result, we need the following lemmas. The first one extends a famous result by Zalcman [22] concerning normal families. 
Lemma 2.1 (see [23]). Let $\mp$ be a family of meromorphic functions on the unit disc satisfying all zeros of functions in Fwhich have multiplicity $\geq p$ and all poles of functions in Fwhich have multiplicity $\geq q$. Let $\alpha$ be a real number satisfying $-q<\alpha<p$. Then $\mathcal{F}$ is not normal at 0 if and only if there exist

(a) a number $0<r<1$,

(b) points $z_{n}$ with $\left|z_{n}\right|<r$,

(c) functions $f_{n} \in \mathcal{F}$,

(d) positive numbers $\rho_{n} \rightarrow 0$

such that $g_{n}(\zeta):=\rho^{-\alpha} f_{n}\left(z_{n}+\rho_{n} \zeta\right)$ converges spherically uniformly on each compact subset of $\mathbb{C}$ to a nonconstant meromorphic function $g(\zeta)$, whose all zeros have multiplicity $\geq p$ and all poles have multiplicity $\geq q$ and order is at most 2 .

Remark 2.2. If $\mp$ is a family of holomorphic functions on the unit disc in Lemma 2.1, then $g(\zeta)$ is a nonconstant entire function whose order is at most 1.

The order of $g$ is defined by using Nevanlinna's characteristic function $T(r, g)$ :

$$
\rho(g)=\lim _{r \rightarrow \infty} \sup \frac{\log T(r, g)}{\log r}
$$

Lemma 2.3 (see [24] or [25]). Let $f(z)$ be a meromorphic function and $c \in \mathbb{C} \backslash\{0\}$. If $f(z)$ has neither simple zero nor simple pole and $f^{\prime}(z) \neq c$, then $f(z)$ is constant.

Lemma 2.4 (see [26]). Let $f(z)$ be a transcendental meromorphic function of finite order in $\mathbb{C}$, with no simple zero; then $f^{\prime}(z)$ assumes every nonzero finite value infinitely often.

Lemma 2.5 (see [3]). Let $f(z)$ be a meromorphic function in $\mathbb{C}$; then

$$
\begin{gathered}
T(r, f) \leq\left(2+\frac{1}{k}\right) N\left(r, \frac{1}{f}\right)+\left(2+\frac{2}{k}\right) \bar{N}\left(r, \frac{1}{f^{(k)}-1}\right)+S(r, f), \\
T(r, f) \leq \bar{N}(r, f)+N\left(r, \frac{1}{f}\right)+\bar{N}\left(r, \frac{1}{f^{(k)}-1}\right)+S(r, f) .
\end{gathered}
$$

Remark 2.6. Both (2.2) and (2.3) are called the Hayman inequality and Milloux inequality, respectively.

\section{Proof of the Results}

Proof of Theorem 1.7. Suppose that $\mathcal{F}$ is not normal in $D$. Then there exists at least one point $z_{0}$ such that $\mathcal{F}$ is not normal at the point $z_{0}$. Without loss of generality we assume that $z_{0}=0$. By Lemma 2.1, there exist points $z_{j} \rightarrow 0$, positive numbers $\rho_{j} \rightarrow 0$, and functions $f_{j} \in \mathcal{F}$ such that

$$
g_{j}(\xi)=\rho_{j}^{1 / 2} f_{j}\left(z_{j}+\rho_{j} \xi\right) \Longrightarrow g(\xi)
$$


locally uniformly with respect to the spherical metric, where $g$ is a nonconstant meromorphic function in $\mathbb{C}$. Moreover, the order of $g$ is no greater than 2 and the poles of $g$ are of multiplicity $\geq 2$.

From (3.1) we know that

$$
\begin{gathered}
g_{j}^{\prime}(\xi)=\rho_{j}^{3 / 2} f_{j}^{\prime}\left(z_{j}+\rho_{j} \xi\right) \Longrightarrow g^{\prime}(\xi), \\
\rho_{j}^{3 / 2}\left(f_{j}^{\prime}\left(z_{j}+\rho_{j} \xi\right)-a f_{j}^{3}\left(z_{j}+\rho_{j} \xi\right)-b\right)=g_{j}^{\prime}(\xi)-a g_{j}^{3}(\xi)-\rho_{j}^{3 / 2} b \Longrightarrow g^{\prime}(\xi)-a g^{3}(\xi)
\end{gathered}
$$

in $\mathbb{C} \backslash \mathbf{S}$ locally uniformly with respect to the spherical metric, where $\mathbf{S}$ is the set of all poles of $g(\xi)$.

If $g^{\prime}-a g^{3} \equiv 0$, then $-(1 / 2)\left(1 / g^{2}\right) \equiv a \xi+c$, where $c$ is a constant. This contradicts with the idea that the poles of $g$ are of multiplicity $\geq 2$. So $g^{\prime}-a g^{3} \not \equiv 0$.

If $g^{\prime}-a g^{3} \neq 0$, then $g^{\prime} / g^{3} \neq a$. Set $g=1 / \varphi$; then $\varphi \varphi^{\prime} \neq-a$. By Lemma 2.3, $\varphi$ is a constant, so $g$ is also a constant which is a contradiction with $g$ being a nonconstant. Hence, $g^{\prime}-a g^{3}$ is a nonconstant meromorphic function and has at least one zero.

Next we prove that $g^{\prime}-a g^{3}$ has just a unique zero. By contraries, let $\xi_{0}$ and $\xi_{0}^{*}$ be two distinct zeros of $g^{\prime}-a g^{3}$, and choose $\delta(>0)$ small enough such that $D\left(\xi_{0}, \delta\right) \cap D\left(\xi_{0}^{*}, \delta\right)=\phi$, where $D\left(\xi_{0}, \delta\right)=\left\{\xi:\left|\xi-\xi_{0}\right|<\delta\right\}$ and $D\left(\xi_{0}^{*}, \delta\right)=\left\{\xi:\left|\xi-\xi_{0}^{*}\right|<\delta\right\}$. From (3.3), by Hurwitz's theorem, there exist points $\xi_{j} \in D\left(\xi_{0}, \delta\right), \xi_{j}^{*} \in D\left(\xi_{0}^{*}, \delta\right)$ such that for sufficiently large $j$

$$
\begin{aligned}
& f_{j}^{\prime}\left(z_{j}+\rho_{j} \xi_{j}\right)-a f_{j}^{3}\left(z_{j}+\rho_{j} \xi_{j}\right)-b=0, \\
& f_{j}^{\prime}\left(z_{j}+\rho_{j} \xi_{j}^{*}\right)-a f_{j}^{3}\left(z_{j}+\rho_{j} \xi_{j}^{*}\right)-b=0 .
\end{aligned}
$$

By the hypothesis that, for each pair of functions $f$ and $g$ in $\mathcal{F}, f^{\prime}-a f^{3}$ and $g^{\prime}-a g^{3}$ share $b$ in $D$, we know that, for any positive integer $m$

$$
\begin{gathered}
f_{m}^{\prime}\left(z_{j}+\rho_{j} \xi_{j}\right)-a f_{m}^{3}\left(z_{j}+\rho_{j} \xi_{j}\right)-b=0 \\
f_{m}^{\prime}\left(z_{j}+\rho_{j} \xi_{j}^{*}\right)-a f_{m}^{3}\left(z_{j}+\rho_{j} \xi_{j}^{*}\right)-b=0 .
\end{gathered}
$$

Fix $m$, take $j \rightarrow \infty$, and note that $z_{j}+\rho_{j} \xi_{j} \rightarrow 0, z_{j}+\rho_{j} \xi_{j}^{*} \rightarrow 0$; then $f_{m}^{\prime}(0)-a f_{m}^{3}(0)-b=0$. Since the zeros of $f_{m}^{\prime}-a f_{m}^{3}-b$ have no accumulation point,

$$
z_{j}+\rho_{j} \xi_{j}=0, \quad z_{j}+\rho_{j} \xi_{j}^{*}=0
$$

Hence, $\xi_{j}=-\left(z_{j} / \rho_{j}\right), \xi_{j}^{*}=-\left(z_{j} / \rho_{j}\right)$. This contradicts with $\xi_{j} \in D\left(\xi_{0}, \delta\right), \xi_{j}^{*} \in D\left(\xi_{0}^{*}, \delta\right)$, and $D\left(\xi_{0}, \delta\right) \cap D\left(\xi_{0}^{*}, \delta\right)=\phi$. So $g^{\prime}-a g^{3}$ has just a unique zero, which can be denoted by $\xi_{0}$.

Set $g=1 / \varphi$ again; then $g^{\prime}-a g^{3}=-\left(\varphi^{\prime} \varphi+a\right) / \varphi^{3}$. So $\left(\varphi^{\prime} \varphi+a\right) / \varphi^{3}$ has only a unique zero $\xi_{0}$. Therefore, $\xi_{0}$ is a multiple pole of $\varphi$, or else a zero of $\varphi^{\prime} \varphi+a$. If $\xi_{0}$ is a multiple pole of $\varphi$, since $\left(\varphi^{\prime} \varphi+a\right) / \varphi^{3}$ has only one zero $\xi_{0}$, then $\varphi^{\prime} \varphi+a \neq 0$. By Lemma 2.3 again, $\varphi$ is a constant which contradicts with the idea that $g$ is a nonconstant.

So $\varphi$ has no multiple pole and $\varphi^{\prime} \varphi+a$ has just a unique zero $\xi_{0}$. By Lemma $2.3, \varphi$ is not any transcendental function. 
If $\varphi$ is a nonconstant polynomial, then $\varphi^{\prime} \varphi+a=A\left(\xi-\xi_{0}\right)^{l}$, where $A$ is a nonzero constant and $l$ is a positive integer because the poles of $g$ are of multiplicity $\geq 2$. So the zeros of $\varphi$ are of multiplicity $\geq 2$, and thus, $l \geq 3$. Set $\psi=(1 / 2) \varphi^{2}$; then $\psi^{\prime}=A\left(\xi-\xi_{0}\right)^{l}-a$ and $\psi^{\prime \prime}=A l\left(\xi-\xi_{0}\right)^{l-1}$. Note that the zeros of $\psi$ are of multiplicity $\geq 4$. But $\psi^{\prime \prime}$ has only one zero $\xi_{0}$, and so $\psi$ has only the same zero $\xi_{0}$. Hence $\psi^{\prime}\left(\xi_{0}\right)=0$ which contradicts with $\psi^{\prime}\left(\xi_{0}\right)=a \neq 0$. Therefore $\varphi$ and $\psi$ are rational functions which are not polynomials and $\psi^{\prime}+a$ has just a unique zero $\xi_{0}$.

Next we prove that there exists no rational function such as $\psi$. Noting that $\psi=(1 / 2) \varphi^{2}$, $\varphi$ has no multiple pole, and the zeros of $\varphi$ are of multiplicity $\geq 2$, we can set

$$
\psi(\xi)=A \frac{\left(\xi-\xi_{1}\right)^{m_{1}}\left(\xi-\xi_{2}\right)^{m_{2}} \cdots\left(\xi-\xi_{s}\right)^{m_{s}}}{\left(\xi-\eta_{1}\right)^{2}\left(\xi-\eta_{2}\right)^{2} \cdots\left(\xi-\eta_{t}\right)^{2}}
$$

where $A$ is a nonzero constant, $s \geq 1, t \geq 1$, and $m_{i} \geq 4(i=1,2, \ldots, s)$. For stating briefly, denote

$$
m=m_{1}+m_{2}+\cdots+m_{s} \geq 4 s
$$

From (3.7),

$$
\psi^{\prime}(\xi)=\frac{A\left(\xi-\xi_{1}\right)^{m_{1}-1}\left(\xi-\xi_{2}\right)^{m_{2}-1} \cdots\left(\xi-\xi_{s}\right)^{m_{s}-1} h(\xi)}{\left(\xi-\eta_{1}\right)^{3}\left(\xi-\eta_{2}\right)^{3} \cdots\left(\xi-\eta_{t}\right)^{3}}=\frac{p_{1}(\xi)}{q_{1}(\xi)}
$$

where

$$
\begin{gathered}
h(\xi)=(m-2 t) \xi^{s+t-1}+a_{s+t-2} \xi^{s+t-2}+\cdots+a_{0} \\
p_{1}(\xi)=A\left(\xi-\xi_{1}\right)^{m_{1}-1}\left(\xi-\xi_{2}\right)^{m_{2}-1} \cdots\left(\xi-\xi_{s}\right)^{m_{s}-1} h(\xi) \\
q_{1}(\xi)=\left(\xi-\eta_{1}\right)^{3}\left(\xi-\eta_{2}\right)^{3} \cdots\left(\xi-\eta_{t}\right)^{3}
\end{gathered}
$$

are polynomials. Since $\psi^{\prime}(\xi)+a$ has only a unique zero $\xi_{0}$, set

$$
\psi^{\prime}(\xi)+a=\frac{B\left(\xi-\xi_{0}\right)^{l}}{\left(\xi-\eta_{1}\right)^{3}\left(\xi-\eta_{2}\right)^{3} \cdots\left(\xi-\eta_{t}\right)^{3}},
$$

where $B$ is a nonzero constant, and so

$$
\psi^{\prime \prime}(\xi)=\frac{\left(\xi-\xi_{0}\right)^{l-1} p_{2}(\xi)}{\left(\xi-\eta_{1}\right)^{4}\left(\xi-\eta_{2}\right)^{4} \cdots\left(\xi-\eta_{t}\right)^{4}},
$$


where $p_{2}(\xi)=B(l-3 t) \xi^{t}+b_{t-1} \xi^{t-1}+\cdots+b_{0}$ is a polynomial. From (3.9), we also have

$$
\psi^{\prime \prime}(\xi)=\frac{\left(\xi-\xi_{1}\right)^{m_{1}-2}\left(\xi-\xi_{2}\right)^{m_{2}-2} \cdots\left(\xi-\xi_{s}\right)^{m_{s}-2} p_{3}(\xi)}{\left(\xi-\eta_{1}\right)^{4}\left(\xi-\eta_{2}\right)^{4} \cdots\left(\xi-\eta_{t}\right)^{4}}
$$

where $p_{3}(\xi)$ is also a polynomial.

Let $\operatorname{deg}(p)$ denote the degree of a polynomial $p(\xi)$.

From (3.9) and (3.10),

$$
\operatorname{deg}(h) \leq s+t-1, \quad \operatorname{deg}\left(p_{1}\right) \leq m+t-1, \quad \operatorname{deg}\left(q_{1}\right)=3 t .
$$

Similarly from (3.12) and (3.13) and noting (3.14),

$$
\begin{gathered}
\operatorname{deg}\left(p_{2}\right) \leq t \\
\operatorname{deg}\left(p_{3}\right) \leq \operatorname{deg}\left(p_{1}\right)+t-1-(m-2 s) \leq 2 t+2 s-2 .
\end{gathered}
$$

Note that $m_{i} \geq 4(i=1,2, \ldots, s)$. It follows from (3.9) and (3.11) that $\psi^{\prime}\left(\xi_{i}\right)=0(i=$ $1,2, \ldots, s)$ and $\psi^{\prime}\left(\xi_{0}\right)=a \neq 0$. Thus $\xi_{0} \neq \xi_{i}(i=1,2, \ldots, s)$, and then $\left(\xi-\xi_{0}\right)^{l-1}$ is a factor of $p_{3}(\xi)$. Hence we get that $l-1 \leq \operatorname{deg}\left(p_{3}\right)$. Combining (3.12) and (3.13) we also have $m-2 s=$ $\operatorname{deg}\left(p_{2}\right)+l-1-\operatorname{deg}\left(p_{3}\right) \leq \operatorname{deg}\left(p_{2}\right)$. By $(3.15)$ we obtain

$$
m-2 s \leq \operatorname{deg}\left(p_{2}\right) \leq t
$$

Since $m \geq 4 s$, we know by (3.17) that

$$
2 s \leq t
$$

If $l \geq 3 t$, by (3.16), then $3 t-1 \leq l-1 \leq \operatorname{deg}\left(p_{3}\right) \leq 2 t+2 s-2$. Noting (3.18), we obtain $1 \leq 0$, a contradiction.

If $l<3 t$, from (3.9) and (3.11), $\operatorname{deg}\left(p_{1}\right)=\operatorname{deg}\left(q_{1}\right)$. Noting that $\operatorname{deg}\left(p_{1}\right)=m+t-i, 1 \leq$ $i \leq s+t, \operatorname{deg}\left(q_{1}\right)=3 t$, so $m+t-i=3 t, m=2 t+i \neq 2 t$. From (3.10), then $\operatorname{deg}(h)=s+t-1$, and then $\operatorname{deg}\left(p_{1}\right)=m+t-1$. Noting that $\operatorname{deg}\left(q_{1}\right)=3 t, m=2 t+1$. By (3.17), $t \leq 2 s-1$. From (3.18), we obtain $1 \leq 0$, a contradiction.

The proof of Theorem 1.7 is complete.

Proof of Theorem 1.9. Similarly with the proof of Theorem 1.7, we assume that $\mathcal{F}$ is not normal at $z_{0}=0$. Then, by Lemma 2.1 , there are a sequence of complex numbers $z_{j}, z_{j} \rightarrow 0(j \rightarrow \infty)$, a sequence of functions $f_{j} \in \mathcal{F}$, and a sequence of positive numbers $\rho_{j} \rightarrow 0^{+}$such that $g_{j}(\xi)=\rho_{j} f_{j}\left(z_{j}+\rho_{j} \xi\right)$ converges uniformly with respect to the spherical metric to a nonconstant mermorphic function $g(\xi)$ and all zeros and poles of $g(\xi)$ are multiple. Moreover, $g(\xi)$ is of order 2 at most. 
Thus

$$
\begin{gathered}
g_{j}^{\prime}(\xi)=\rho_{j}^{2} f_{j}^{\prime}\left(z_{j}+\rho_{j} \xi\right) \Longrightarrow g^{\prime}(\xi) \\
\rho_{j}^{2}\left(f_{j}^{\prime}\left(z_{j}+\rho_{j} \xi\right)-a f_{j}^{2}\left(z_{j}+\rho_{j} \xi\right)-b\right)=g_{j}^{\prime}(\xi)-a g_{j}^{2}(\xi)-\rho_{j}^{2} b \Longrightarrow g^{\prime}(\xi)-a g^{2}(\xi)
\end{gathered}
$$

also locally uniformly with respect to the spherical metric.

If $g^{\prime}-a g^{2} \equiv 0$, then $-(1 / g) \equiv a \xi+c$ where $c$ is a constant. This contradicts with the idea that the poles of $g$ are multiple. So $g^{\prime}-a g^{2} \not \equiv 0$.

If $g^{\prime}-a g^{2} \neq 0$, then $(1 / g)^{\prime} \neq-a$. By Lemma 2.3, $g$ is a constant which contradicts with our conclusion. Hence, $g^{\prime}-a g^{2}$ is a nonconstant meromorphic function and has at least one zero.

As the same argument in the proof of Theorem 1.7, we obtain that $g^{\prime}-a g^{2}$ has only one distinct zero denoted by $\xi_{0}$.

Set $g=1 / \varphi$; then $g^{\prime}-a g^{2}=-\left(\varphi^{\prime}+a\right) / \varphi^{2}$. So $\left(\varphi^{\prime}+a\right) / \varphi^{2}$ has only a unique zero $\xi_{0}$. Therefore $\xi_{0}$ is a multiple pole of $\varphi$ or a zero of $\varphi^{\prime}+a$. If $\xi_{0}$ is a multiple pole of $\varphi$, since $\left(\varphi^{\prime}+a\right) / \varphi^{2}$ has only one zero $\xi_{0}$, then $\varphi^{\prime}+a \neq 0$. By Lemma 2.3 again, $\varphi$ is a constant, which is a contradiction.

Hence $\varphi$ is an entire function with no simple zero and growth order at most 2 and $\varphi^{\prime}+a$ has just a unique zero $\xi_{0}$. By Lemma 2.4, $\varphi$ is not any transcendental function. Therefore $\varphi$ is a nonconstant polynomial and has the form that $\varphi^{\prime}+a=C\left(\xi-\xi_{0}\right)^{l}$, where $C$ is a nonzero constant and $l$ is a positive integer because the poles of $g$ are of multiplicity $\geq 3$. So the zeros of $\varphi$ are of multiplicity $\geq 3$; thus, $l \geq 2, \varphi^{\prime \prime}=C l\left(\xi-\xi_{0}\right)^{l-1}$. Note that $\varphi^{\prime \prime}$ has only one zero $\xi_{0}$, and so $\varphi$ has only the same zero $\xi_{0}$ too. Hence $\varphi^{\prime}\left(\xi_{0}\right)=0$ which contradicts with $\varphi^{\prime}\left(\xi_{0}\right)=-a \neq 0$.

The proof of Theorem 1.9 is complete.

Proof of Theorem 1.11. Similarly with the proof of Theorem 1.7, we assume that $\mathcal{F}$ is not normal at $z_{0}=0$. Then, by Lemma 2.1, there are a sequence of complex numbers $z_{j}, z_{j} \rightarrow 0(j \rightarrow \infty)$, a sequence of functions $f_{j} \in \mathcal{F}$, and a sequence of positive numbers $\rho_{j} \rightarrow 0^{+}$such that $g_{j}(\xi)=$ $\rho_{j}^{-1} f_{j}\left(z_{j}+\rho_{j} \xi\right)$ converges uniformly with respect to the spherical metric to a nonconstant meromorphic function $g(\xi)$ whose poles are multiple and $g(\xi) \neq 0$. Moreover, $g(\xi)$ is of order 2 at most.

Thus

$$
\begin{gathered}
g_{j}^{\prime}(\xi)=f_{j}^{\prime}\left(z_{j}+\rho_{j} \xi\right) \Longrightarrow g^{\prime}(\xi) \\
g_{j}^{\prime}(\xi)-a \rho_{j} g_{j}(\xi)-b=f_{j}^{\prime}\left(z_{j}+\rho_{j} \xi\right)-a f_{j}\left(z_{j}+\rho_{j} \xi\right)-b \Longrightarrow g^{\prime}(\xi)-b
\end{gathered}
$$

also locally uniformly with respect to the spherical metric. $g^{\prime}-b \not \equiv 0$.

If $g^{\prime}-b \equiv 0$, then $g=b \xi+c$, where $c$ is a constant. This contradicts with $g(\xi) \neq 0$. So 
If $g^{\prime}-b \neq 0$, then by Milloux inequality (2.3) of Lemma 2.5 we have

$$
\begin{aligned}
T(r, g) & \leq \bar{N}(r, g)+N\left(r, \frac{1}{g}\right)+\bar{N}\left(r, \frac{1}{g^{\prime}-b}\right)+S(r, g) \\
& \leq \frac{1}{2} N(r, g)+S(r, g) \\
& \leq \frac{1}{2} T(r, g)+S(r, g) .
\end{aligned}
$$

From (3.23) we know that $g$ is a constant which contradicts with our conclusion. Hence, $g^{\prime}-b$ is a nonconstant meromorphic function and has at least one zero.

As the same argument in the proof of Theorem 1.7, we obtain that $g^{\prime}-b$ has only one distinct zero denoted by $\xi_{0}$. Thus the Hayman inequality (2.2) of Lemma 2.5 implies that $g$ is a rational function of degree 4 at most. Noting that $g \neq 0$ and has no simple pole, we obtain that $g$ has at most two distinct poles. Using the Milloux inequality (2.3) of Lemma 2.5 again we get that $g$ has at most one distinct pole. Hence we can write $g(\xi)=1 /(d \xi+e)^{m}, 2 \leq m \leq 3$, where $d \neq 0$ and $e$ are two finite complex numbers. Simple calculation shows that $g^{\prime}-b$ has at least three distinct zeros. This is impossible.

The proof of Theorem 1.11 is complete.

Proof of Theorem 1.14. Similarly with the proof of Theorem 1.11, we have that (3.22) also holds. Moreover, $g(\xi) \neq 0$ and $g(\xi)$ is of order 2 at most.

If $g^{\prime}-b \equiv 0$, then $g=b \xi+c$, where $c$ is a constant. This contradicts with $g(\xi) \neq 0$.

If $g^{\prime}-b \neq 0$, then by the Hayman inequality (2.2) of Lemma 2.5 we have

$$
\begin{aligned}
T(r, g) & \leq 3 N\left(r, \frac{1}{g}\right)+4 \bar{N}\left(r, \frac{1}{g^{\prime}-b}\right)+S(r, g) \\
& \leq S(r, g) .
\end{aligned}
$$

From (3.24) we know that $g$ is a constant which contradicts with our conclusion.

The proof of Theorem 1.8 is complete.

\section{Acknowledgments}

The authors would like to express their hearty thanks to Professor Qingcai Zhang for supplying them with his helpful reprint. The authors wish to thank the managing editor and referees for their very helpful comments and useful suggestions. This work was completed with the support of the NSF of China (10771220) and Doctorial Point Fund of National Education Ministry of China (200810780002).

\section{References}

[1] C.-C. Yang and H.-X. Yi, Uniqueness Theory of Meromorphic Functions, vol. 557 of Mathematics and Its Applications, Kluwer Academic Publishers, Dordrecht, The Netherlands, 2003.

[2] Y. X. Gu, X. C. Pang, and M. L. Fang, Theory of Normal Family and Its Applications, Science Press, Beijing, China, 2007. 
[3] W. K. Hayman, Meromorphic Functions, Oxford Mathematical Monographs, Clarendon Press, Oxford, UK, 1964.

[4] L. Yang, Value Distribution Theory, Springer, Berlin, Germany, 1993.

[5] W. Bergweiler, "Bloch's principle," Computational Methods and Function Theory, vol. 6, no. 1, pp. 77-108, 2006.

[6] L. A. Rubel, "Four counterexamples to Bloch's principle," Proceedings of the American Mathematical Society, vol. 98, no. 2, pp. 257-260, 1986.

[7] D. Drasin, "Normal families and the Nevanlinna theory," Acta Mathematica, vol. 122, pp. 231-263, 1969.

[8] W. Schwick, "Normality criteria for families of meromorphic functions," Journal d'Analyse Mathématique, vol. 52, pp. 241-289, 1989.

[9] X. Pang and L. Zalcman, "Normal families and shared values," The Bulletin of the London Mathematical Society, vol. 32, no. 3, pp. 325-331, 2000.

[10] X. Pang and L. Zalcman, "Normality and shared values," Arkiv för Matematik, vol. 38, no. 1, pp. 171$182,2000$.

[11] Q. Zhang, "Normal families of meromorphic functions concerning shared values," Journal of Mathematical Analysis and Applications, vol. 338, no. 1, pp. 545-551, 2008.

[12] W. K. Hayman, Research Problems in Function Theory, The Athlone Press of University of London, London, UK, 1967.

[13] X. C. Pang, "On normal criterion of meromorphic functions," Science in China A, vol. 33, no. 5, pp. 521-527, 1990.

[14] H. H. Chen and M. L. Fang, "On a theorem of Drasin," Advances in Mathematics, vol. 20, p. 504, 1991 (Chinese).

[15] Y. S. Ye, "A new normality criterion and its application," Chinese Annals of Mathematics A, vol. 12, supplement, pp. 44-49, 1991.

[16] H. H. Chen and Y. X. Gu, "Improvement of Marty's criterion and its application," Science in China A, vol. 36, no. 6, pp. 674-681, 1993.

[17] S. Y. Li, “On normality criterion of a class of the functions," Journal of Fujian Normal University, vol. 2, pp. 156-158, 1984.

[18] X. J. Li, "Proof of Hayman's conjecture on normal families," Science in China A, vol. 28, no. 6, pp. 596-603, 1985.

[19] J. K. Langley, "On normal families and a result of Drasin," Proceedings of the Royal Society of Edinburgh A, vol. 98, no. 3-4, pp. 385-393, 1984.

[20] L. Zalcman, “On some questions of Hayman," . In press.

[21] M. Fang and W. Yuan, "On the normality for families of meromorphic functions," Indian Journal of Mathematics, vol. 43, no. 3, pp. 341-351, 2001.

[22] L. Zalcman, "A heuristic principle in complex function theory," The American Mathematical Monthly, vol. 82, no. 8, pp. 813-817, 1975.

[23] L. Zalcman, "Normal families: new perspectives," Bulletin of American Mathematical Society, vol. 35, no. 3, pp. 215-230, 1998.

[24] W. Bergweiler and X. Pang, "On the derivative of meromorphic functions with multiple zeros," Journal of Mathematical Analysis and Applications, vol. 278, no. 2, pp. 285-292, 2003.

[25] Y. Wang and M. Fang, "Picard values and normal families of meromorphic functions with multiple zeros," Acta Mathematica Sinica, vol. 14, no. 1, pp. 17-26, 1998.

[26] W. Bergweiler and A. Eremenko, "On the singularities of the inverse to a meromorphic function of finite order," Revista Matemática Iberoamericana, vol. 11, no. 2, pp. 355-373, 1995. 


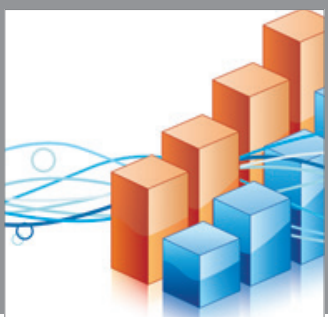

Advances in

Operations Research

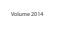

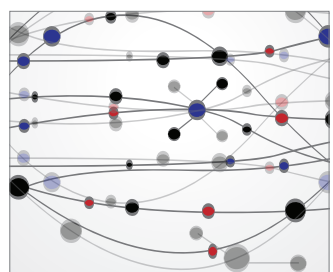

\section{The Scientific} World Journal
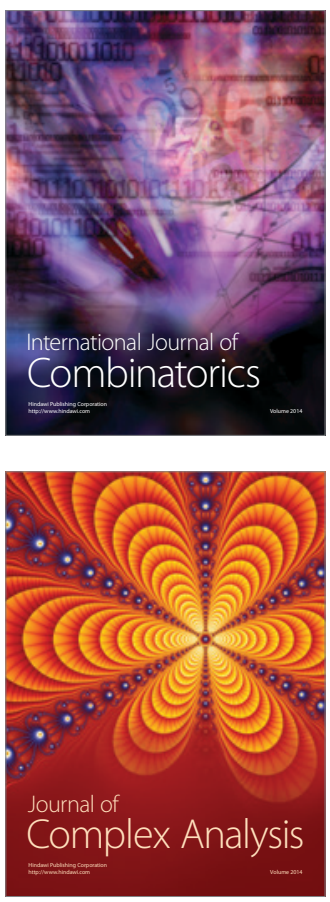

International Journal of

Mathematics and

Mathematical

Sciences
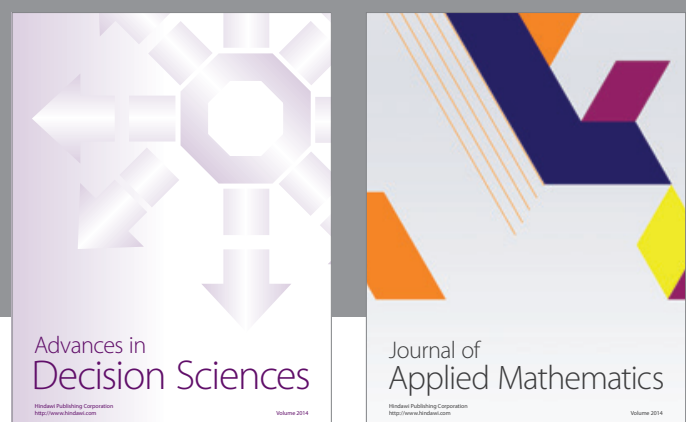

Journal of

Applied Mathematics
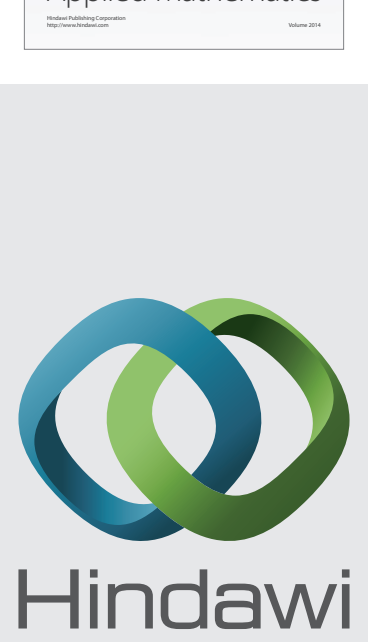

Submit your manuscripts at http://www.hindawi.com
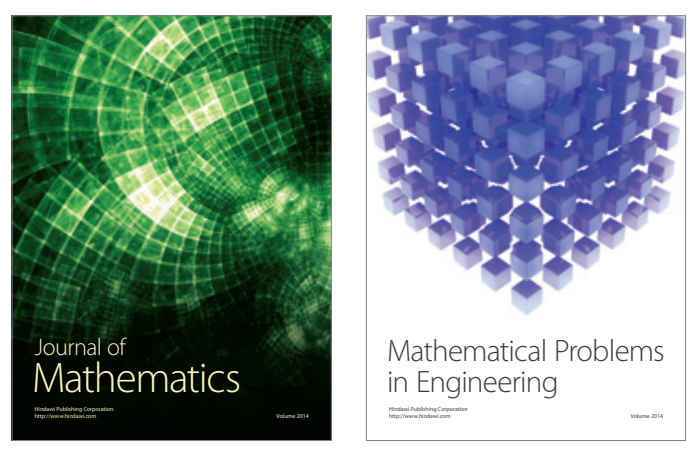

Mathematical Problems in Engineering
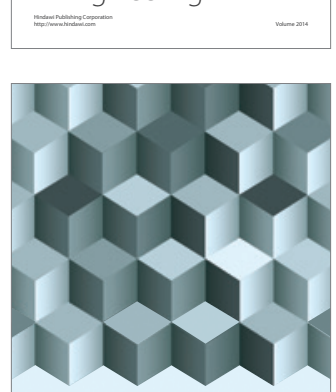

Journal of

Function Spaces
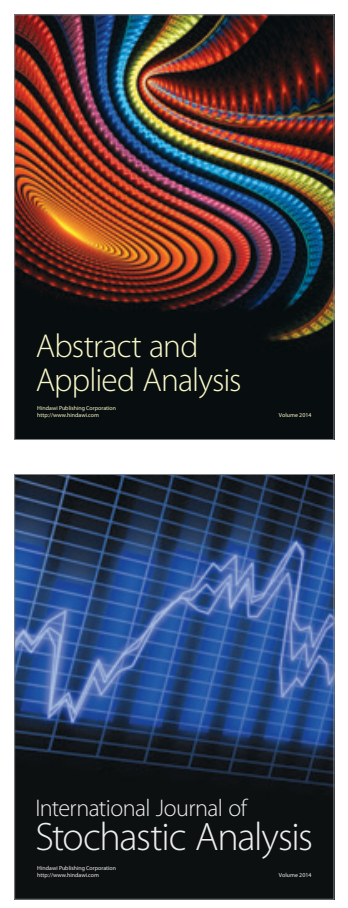

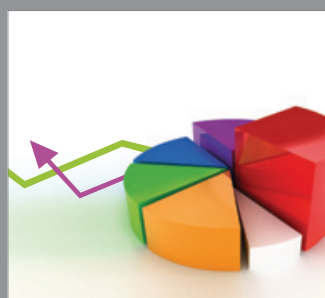

ournal of

Probability and Statistics

Promensencen
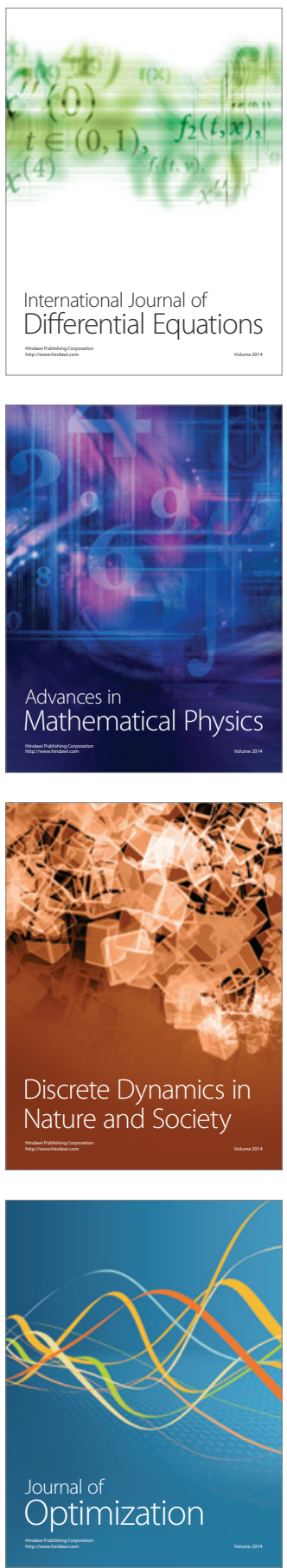